\title{
Os anticoncepcionais orais como fator de risco cardiovascular: uma revisão narrativa
}

\author{
Oral contraceptives as a cardiovascular risk factor: a narrative review
}

Anticonceptivos orales como factor de riesgo cardiovascular: una revisión narrativa

Thiago Mendes dos Santos ${ }^{1 *}$, Manuela de Matos Costa de Menezes ${ }^{1}$, Mariana Pereato Fernandes ${ }^{2}$, Giovana Lopes Nadais ${ }^{3}$, Maria Fernanda de Almeida Gomes ${ }^{3}$, Serginara Cristina Flexa Pereira da Silva ${ }^{4}$, Rebeca Fernandes de Azevedo Dantas ${ }^{5}$, Maria Eduarda Barillari Cano ${ }^{6}$, Bruno Augusto Lopes ${ }^{7}$.

\section{RESUMO}

Objetivo: Revisar e analisar a relação da anticoncepção hormonal como fator de risco cardiovascular. Revisão bibliográfica: $\mathrm{Na}$ tentativa de reduzir os efeitos colaterais do uso de anticoncepcionais hormonais, foram criadas novas formulações com alterações da dose do estrógeno e formulações baseadas em progesterona. O uso de anticoncepcionais combinados aumenta o risco tanto de tromboses venosas quanto arteriais. Há evidências de alterações pressóricas associadas ao uso de anticoncepcionais orais combinados (ACOs). Nota-se uma íntima relação entre a ingesta de pílulas contraceptivas e a alteração das taxas lipídicas corporais, o que, de forma indireta, potencializa o risco cardiovascular. O uso de contraceptivos orais está relacionado a tromboses arteriais que, por sua vez, podem cursar com infarto agudo do miocárdio (IAM). $O$ risco geral de trombose arterial é 1,6x maior em mulheres que fazem uso de contraceptivos orais. Considerações finais: $O$ uso do estrogênio exógeno contido nos ACOs, principalmente em altas doses, é um fator preponderante para o desenvolvimento de doenças cardiovasculares. É crucial que ocorra a conscientização acerca da automedicação potencialmente perigosa no uso dos anticoncepcionais hormonais e que os profissionais de saúde prescrevam esse método contraceptivo de forma individualizada, visando evitar desfechos desfavoráveis.

Palavras-chave: Anticoncepcionais, Fatores de risco, Doenças cardiovasculares.

\begin{abstract}
Objective: Review and analyze the relationship of hormonal contraception as a cardiovascular risk factor. Bibliographic review: In an attempt to reduce the side effects of the use of hormonal contraceptives, new formulations were created with changes in the dose of estrogen and formulations based on progesterone. The use of combined contraceptives increases the risk of both venous and arterial thrombosis. There is evidence of blood pressure changes associated with the use of combined oral contraceptives (ACOs). There is a close relationship between the intake of contraceptive pills and changes in body lipid levels, which, indirectly, potentiates cardiovascular risk. The use of oral contraceptives is related to arterial thrombosis, which, in turn, can lead to acute myocardial infarction (AMI). The overall risk of arterial thrombosis is $1.6 \mathrm{x}$ greater in women who use oral contraceptives. Final considerations: The use of exogenous estrogen contained in OCs, especially in high doses, is a major factor in the development of cardiovascular disease. It is crucial to raise awareness about potentially dangerous self-medication in the use of hormonal contraceptives and for health professionals to prescribe this contraceptive method individually, in order to avoid unfavorable outcomes.
\end{abstract}

Keywords: Contraceptives, Risk factors, Cardiovascular diseases.

1 Universidade de Vassouras (UV), Vassouras - RJ. *E-mail: thiagomedvassouras@gmail.com

2 Faculdade de Medicina de Campos (FMC), Campos dos Goytacazes - RJ.

${ }^{3}$ Universidade do Grande Rio (Unigranrio), Rio de Janeiro - RJ.

${ }^{4}$ Centro Universitário do Estado do Pará (CESUPA), Belém - PA.

${ }^{5}$ Universidade Estácio de Sá (UNESA), Rio de Janeiro - RJ.

${ }^{6}$ Faculdade de Ciências Médicas e da Saúde de Juiz de Fora (FCMS), Juiz de Fora - MG.

${ }^{7}$ Hospital Municipal Miguel Couto (HMMC), Rio de Janeiro - RJ. 


\section{RESUMEN}

Objetivo: Revisar y analizar la relación de la anticoncepción hormonal como factor de riesgo cardiovascular. Revisión bibliográfica: En un intento por reducir los efectos secundarios del uso de anticonceptivos hormonales, se crearon nuevas formulaciones con cambios en la dosis de estrógeno y formulaciones a base de progesterona. El uso de anticonceptivos combinados aumenta el riesgo de trombosis arterial y venosa. Existe evidencia de cambios en la presión arterial asociados con el uso de anticonceptivos orales combinados (ACO). Existe una estrecha relación entre la ingesta de píldoras anticonceptivas y los cambios en los niveles de lípidos corporales, lo que, indirectamente, potencia el riesgo cardiovascular. El uso de anticonceptivos orales está relacionado con la trombosis arterial que, a su vez, puede derivar en un infarto agudo de miocardio (IAM). El riesgo general de trombosis arterial es 1,6 veces mayor en las mujeres que usan anticonceptivos orales. Consideraciones finales: El uso de estrógenos exógenos contenidos en los $\mathrm{AO}$, especialmente en dosis altas, es un factor importante en el desarrollo de enfermedades cardiovasculares. Es fundamental crear conciencia sobre la automedicación potencialmente peligrosa en el uso de anticonceptivos hormonales y que los profesionales de la salud prescriban este método anticonceptivo de forma individual, para evitar resultados desfavorables.

Palabras clave: Anticonceptivos, Factores de riesgo, Enfermedades cardiovasculares.

\section{INTRODUÇÃO}

A habilidade de controlar a fertilidade utilizando efetivamente a contracepção é um componente essencial da medicina preventiva. O primeiro anticoncepcional esteroidal foi aprovado em 1960 e se tornou popular pela facilidade do seu uso e criação de uma sensação de empoderamento e liberdade no público feminino da época. Desde a sua criação, esse método de contracepção tem aumentado progressivamente a sua acessibilidade e popularidade. Após sua aprovação para consumo, a preocupação com os efeitos colaterais se tornou uma discussão frequente que exigiu formulações mais seguras (OLIVEIRA RPC e TREVISAN M, 2021).

Os anticoncepcionais hormonais orais passaram por mudanças consideráveis em sua composição, tendo alterações em seu tipo de esteroide e dose. A primeira geração de anticoncepcionais orais continha mestranol, que foi substituído progressivamente por etinil estradiol (EE). As doses, então, foram progressivamente diminuídas passando de $50 \mathrm{mcg}$ para 30 e $20 \mathrm{mcg}$ (SITRUK-WARE R, 2020).

Os anticoncepcionais hormonais são os métodos contraceptivos mais usados pelas mulheres, sendo a principal forma de contracepção na Europa e na América do Norte. Essas regiões, possuem uma adesão aproximada de $17,8 \%$ e são utilizados por mais de 150 milhões de mulheres internacionalmente (ENEA C et al., 2021; OEDINGEN C, et al., 2018). A nível nacional, a porcentagem feminina adepta a algum método anticoncepcional está em torno de $80,6 \%$, sendo $24,7 \%$ das mulheres utilizadoras de pílulas orais hormonais (MINISTÉRIO DA SAÚDE, 2016). Uma grande justificativa para o seu uso difundido são os seus efeitos terapêuticos, além da prevenção de uma gestação indesejada, diminuição da síndrome pré-menstrual e para o controle de miomas uterinos, por exemplo (ROMER T, 2019).

As diretrizes elaboradas pelo American College of Obstetrician and Gynecologists (ACOG) abordam a necessidade do uso dos anticoncepcionais hormonais em mulheres acima de 35 anos como prática fundamental para prevenção de gravidez. No entanto, também ressaltam o potencial risco de prescrição dessas medicações, sobretudo na presença de comorbidades e determinados estilos de vida, como diabetes, hipertensão, obesidade e tabagismo. Soma-se a isso o risco de potencialização de tais condições clínicas e desenvolvimento de patologias cardiovasculares, posto que os hormônios presentes nas formulações dessas pílulas não conferem proteção cardiovascular (CHO MK, 2018; RÖMER T, 2019). Essa questão se relaciona com o fato de os componentes hormonais das pílulas serem responsáveis pela aceleração da formação de coágulos e pela vasoconstrição (BRITTON LE, et al., 2020).

Em 2015, foram atualizados os critérios de elegibilidade dos anticoncepcionais de alta efetividade, definindo recomendações que relacionam critérios de saúde da mulher e o uso de anticoncepcionais reversíveis. O problema surge quando o tratamento não é individualizado para cada mulher de maneira que 
o torna como fator de risco cardiovascular - acidente vascular encefálico (AVE), infarto, trombose -, posto que os hormônios presentes nesse tipo de medicação potencializam quadros de vasoconstrição sanguínea, além de corroborar uma formação de coágulos de forma mais rápida, fato que prejudica a circulação sanguínea a nível (BRITTON LE, et al., 2020).

Estudos recentes da Women's Health Initiative (WHI) comprovam a íntima relação entre o surgimento e a perpetuação de efeitos pró trombóticos e risco de tromboembolismo venoso cardiovascular (TEV) com a utilização dos anticoncepcionais orais (MANSON JE, et al., 2003). Pesquisas recentes revelam que mulheres que usam pílulas contraceptivas são, aproximadamente, quatro vezes mais susceptíveis ao desenvolvimento de TEV do que mulheres que não as utilizam (SITRUK-WARE R, 2020). Nesse sentido, as doenças cardiovasculares como o infarto agudo do miocárdio são as principais causas de óbitos na população feminina (MINISTÉRIO DA SAÚDE, 2018).

Os componentes hormonais das pílulas levam a uma variação do perfil lipídico, com destaque para a redução dos níveis de HDL. Ademais, há elevação de pressão sanguínea em mulheres previamente normotensas, além de alteração à tolerância à glicose e aumento dos marcadores inflamatórios como a proteína $C$ reativa. Tais alterações são de suma importância para a saúde da mulher, principalmente ao uso difundido de ACO e iminência de efeitos colaterais (FERREIRA LF, et al., 2019; FERREIRA BBR e DA PAIXÃO JÁ, 2021; RAHHAL A, et al., 2020).

O objetivo do presente estudo é analisar a relação da anticoncepção hormonal como fator de risco cardiovascular.

\section{REVISÃO BIBLIOGRÁFICA}

A associação entre os potenciais fatores de risco cardiovasculares e o uso de anticoncepcionais como método contraceptivo contribuiu para que a Organização Mundial da Saúde (OMS) publicasse critérios de elegibilidade para o seu uso. Tais critérios se baseavam no elevado risco de doenças cardiovasculares e tromboembólicas, no qual o risco proporcionado pelo uso dos hormônios disponíveis na medicação seria maior do que o benefício esperado (SONOU A, et al., 2018).

Os anticoncepcionais combinados consistem na associação entre um estrogênio (em geral, o etinilestradiol) e um progestagênio. Os contraceptivos hormonais são classificados como pílulas de alta ou baixa dose (em relação a dose estrogênica) e como primeira, segunda ou terceira geração (em relação ao progestagênio da composição). As gerações foram originadas ao longo dos anos devido às altas taxas de eventos cardiovasculares por fenômenos tromboembólicos (IAM, AVC) desde a introdução dos ACOs no mercado, em 1960. Em 1974, com o aparecimento da segunda geração, as taxas de TEV diminuíram consideravelmente. $O$ mesmo ocorreu na década de $90 \mathrm{com}$ as pílulas de terceira geração. Tal fenômeno continua ocorrendo com a introdução de doses cada vez mais baixas de estrogênio (FINOTTI M, 2015).

Os de primeira geração são compostos por noretisterona associada a $50 \mathrm{mcg}$ de estrogênio. Quando as doses de etinilestradiol são menores $(35,30$ ou $20 \mathrm{mcg}$ ) e associadas ao levonorgestrel ou norgestrel (progestágenos), são chamadas de pílulas de segunda geração. As de terceira geração são compostas por doses ainda menores de etinilestradiol $(\leq 30 \mathrm{mcg}$ ) associadas ao desogestrel ou gestodeno. Atualmente são encontradas pílulas que contêm apenas $15 \mathrm{mcg}$ de etinilestradiol (FINOTTI M, 2015).

$\mathrm{Na}$ tentativa de reduzir os efeitos colaterais do uso de anticoncepcionais hormonais, foram criadas novas formulações com alterações da dose do estrógeno e formulações baseadas em progesterona. As formulações baseadas somente em progesterona não apresentam o mesmo risco de complicações vasculares quanto os que contêm estrógeno (LOPEZ LM, et al., 2019). Em comparação com pacientes que não utilizam anticoncepcional, pesquisas não demonstram que o uso de anticoncepcionais baseados somente em progesterona tenha aumentado o risco de tromboembolismo venoso (HUGON-RODIN J, et al., 2017). Alguns pontuam a superioridade da anticoncepção baseada em progesterona, principalmente em mulheres no período pós-parto, no qual se necessita de uma forma de anticoncepção segura que não interfira na lactação e no risco para tromboembolismo (ANITA M e SHARON C, 2020). 


\section{Tromboembolismo}

A trombose é caracterizada por uma modificação no sistema circulatório resultante do desenvolvimento de trombos na corrente sanguínea, provenientes da união dos elementos da Tríade de Virchow: lesão na parede do vaso sanguíneo, estagnação do sangue no interior da veia e coagulação e hipercoagulabilidade. Dessa forma, esses componentes podem implicar em uma agregação plaquetária, fato que estimula uma série de reações responsáveis pela coagulação sanguínea (CHARLO PB, et al., 2020).

Os trombos podem situar-se em qualquer parte da circulação e, quando se movem, podem alojar-se à distância, fato que leva a uma iminente TEV. A TEV define-se pela ocorrência simultânea da trombose venosa profunda (TVP) e da embolia pulmonar (EP). A primeira citada caracteriza-se por formação de coágulos em veias mais profundas, sobretudo as localizadas em membros inferiores. A EP, por sua vez, é definida como obstrução arterial nos pulmões (ALMEIDA APF e ASSIS MM, 2017).

O estrogênio exógeno - um dos hormônios presentes na composição dos anticoncepcionais - apresenta potenciais riscos de TEV e ação pró-trombótica devido a elevação da protrombina e redução da antitrombina II, o que potencializa a conversão do fibrinogênio em fibrina, sendo este processo essencial para a formação do coágulo (RIBEIRO CCM, et al., 2018). Nesse sentido, devido ao estrogênio presente na formulação das pílulas anticoncepcionais, sobretudo em elevadas doses, mulheres com tendência a desenvolver distúrbios cardiovasculares apresentam maior propensão de desenvolver um quadro de trombose. Vale destacar, ainda, que o estrogênio presente nas pílulas anticoncepcionais potencializa a vasoconstrição por meio do sistema renina-angiotensina, o que pode duplicar ou, até mesmo, quadruplicar as chances de desenvolvimento de uma trombose (RIBEIRO CCM, et al., 2018).

Estudos realizados demonstraram que as pílulas com concentração menor de $50 \mathrm{mcg}$ de etinilestradiol (principal estrógeno endógeno nos humanos) aumenta 4 vezes o risco de desenvolvimento de TEV quando comparado com mulheres que não as usavam. Ademais, quando as concentrações ultrapassam $50 \mathrm{mcg}$ desse hormônio sintético, há elevação de 10 vezes da probabilidade de aparição do distúrbio circulatório em pauta (LOPES LM, et al., 2019). Outra pesquisa a respeito dessa temática foi realizada na população feminina da Dinamarca, concluindo que, de 10.000 mulheres submetidas ao estudo, 6,29\% que utilizavam anticoncepção hormonal de forma crônica apresentaram elevado risco de trombose arterial, sendo uma taxa 3 vezes superior à de mulheres não usuárias dessas pílulas orais (LIMA ACS, et al., 2017).

$O$ uso de anticoncepcionais combinados é contraindicado em mulheres com história prévia de trombose ou presença de fatores de risco (GRAY B, et al., 2018). O risco de trombose arterial não variou entre as diferentes gerações de progestinas, mas o risco relativo aumentou com o aumento de estrogênio (RAHHAL A, et al., 2020).

Os contraceptivos estão mais relacionados à ocorrência de tromboembolismo venoso, entretanto podem ocorrer tromboses arteriais. A associação entre ACO e tromboses arteriais ainda não está bem estabelecida, mas podem ocorrer principalmente em mulheres já com fatores de risco cardiovasculares (tabagismo, hipertensão arterial, diabetes e hipercolesterolemia), podendo ser causa de um IAM. As causas de IAM nas usuárias de $A C O$ são trombóticas e não ateroscleróticas, podendo ser atribuídas aos efeitos pró-trombóticos dos ACO (RAHHAL A, et al., 2020). São necessários mais estudos e pesquisas para o melhor entendimento da relação $A C O$ e tromboses arteriais.

\section{Hipertensão arterial}

Há evidências de alterações pressóricas associadas ao uso de anticoncepcionais orais combinados (AOCs) (OMS, 2018). Estudos demonstram que o seu uso aumenta o risco de eventos vasculares em hipertensas ou naquelas cuja pressão arterial não foi aferida antes do início do uso. Estas alterações seriam causadas pela presença do estrogênio exógeno na circulação sanguínea (independentemente da concentração), a qual ativaria os níveis do sistema renina-angiotensina-aldosterona, com consequente retenção de água e sódio (RIBEIRO C, et al., 2018). Assim, mulheres hipertensas não tratadas ou com controle inadequado não devem fazer uso de AOCs. Vale ressaltar que o antecedente de hipertensão na gravidez não contraindica o uso dessa medicação, mas exige um acompanhamento médico adequado (OMS, 2018). 


\section{Diabetes mellitus}

Diversas pesquisas já associam o uso de ACO hormonais com alterações no metabolismo dos carboidratos, sendo essas alterações a diminuição da tolerância à glicose e o aumento a resistência insulínica. Consequentemente, tais mudanças são um fator de risco para o desenvolvimento de Diabetes Mellitus tipo 2 (DM) sendo o ACO um acelerador para doenças do sistema cardiovascular. Uma explicação seria a influência do estrógeno nos receptores de insulina, com deterioração do metabolismo dos carboidratos e diminuição da sensibilidade insulínica. Por isso, as complicações metabólicas se correlacionam mais fortemente com os ACO contendo estrogênio (FERREIRA LF, et al., 2019; LOPEZ LM, et al., 2019). Entretanto, estudos mais recentes feitos em mulheres não diabéticas ainda pontuam que não existem pesquisas humanas suficientes para confirmar essa correlação (LOPEZ LM, et al., 2019).

Há estudos sobre o antagonismo entre os ACO e os hipoglicemiantes orais, diminuindo os efeitos terapêuticos desses fármacos nas pacientes portadoras de DM em tratamento, que, em alguns casos em que não ocorra a correta associação, pode se ter como consequência quadros de hipoglicemia. Ademais, pode reduzir também as repercussões terapêuticas da insulina, desenvolvendo cenários de hiperglicemia. Dos componentes envolvidos na formação da $\mathrm{DM}$, o glucagon é o único que não tem sua eficácia diminuída pelo uso de anticoncepcionais orais (EVANGELISTA D, et al., 2014).

\section{Perfil lipídico}

Nota-se uma íntima relação entre a ingesta de pílulas contraceptivas e a alteração das taxas lipídicas corporais, o que, de forma indireta, potencializa o risco cardiovascular (LEITE C, 2020). Um estudo realizado com 154 mulheres revela que as usuárias de ACO hormonal apresentam maiores taxas nos níveis de triglicerídeos (TGD), colesterol total (CT), proteína $C$ reativa de Alta sensibilidade (PCR) e lipoproteína de alta densidade (HDL-C) quando comparadas às que não usam. (GUEDES JVN, 2018). Ademais, a elevação dos níveis de TGD conferidos pelos anticoncepcionais orais podem levar a uma potencialização de processos inflamatórios e hipercoagulabilidade pela relação desses lipídeos com dímero-D e PCR. (GIALERAKI A, et al., 2018).

\section{Acidente vascular encefálico}

A literatura ainda é incipiente e controversa quanto à dosagem de estrogênio dos ACO e sua relação com o risco de acidente vascular encefálico (AVE). Estudos atuais demonstram que há aumento da sua incidência com pílulas de estrogênio cuja dosagem é superior a $50 \mathrm{mcg}$. Adicionalmente, uma revisão que analisou 1.298 publicações demonstrou que pacientes em uso de contracepção com pílulas hormonais combinadas não apresentam risco de acidente vascular encefálico hemorrágico. Quanto ao risco de AVE isquêmico, o mesmo foi elevado em mulheres que utilizaram formulações com mais de 50 microgramas de estrogênio, o que representa uma parcela reduzida das prescrições atuais (LIMA ACS, et al., 2017).

Por outro lado, em uma coorte que analisou mais de 80.000 usuárias em uso dessa medicação, o risco de AVE ocorria apenas em pílulas com estrogênio em concentração maior ou igual a $30 \mathrm{mcg}$ por comprimido, além do maior risco também estar associado à idade mais avançada (entre 35 e 55 anos) (ROACH REJ, et al., 2015).

\section{Infarto agudo do miocárdio}

O uso de contraceptivos orais está relacionado a tromboses arteriais que, por sua vez, podem cursar com IAM. Esse efeito colateral pode ter maior ou menor risco a depender da geração do medicamento. Devido aos relatos de trombose coronariana associada ao uso de contraceptivos orais hormonais, foram realizadas modificações nas formulações visando mitigar esse impacto. Os ACOs de terceira geração têm efeito positivo no perfil lipídico (aumento do HDL) e, por isso, protegem contra o infarto do miocárdio (JUREMA K e JUREMA $H, 2021)$.

O risco geral de trombose arterial é 1,6x maior em mulheres que fazem uso de contraceptivos orais. 0 risco não variou dependendo do tipo de progestágeno, entretanto descobriu-se que era $2 x$ maior em mulheres 
que fazem uso com doses mais altas de estrogênio. Dessa maneira, ao longo do tempo os contraceptivos orais foram tendo a dosagem de estrogênio diminuída a fim de diminuir as complicações trombóticas. Os anticoncepcionais orais de terceira geração têm menor quantidade de estrogênio, fato que diminui o risco de trombose. Dessa forma, contraceptivos exclusivos de progestágeno foram considerados seguros, pois 0 estrógeno componente dos contraceptivos orais combinados é o responsável por alterações trombogênicas e pelas alterações em fatores de coagulação que favorecem a formação de trombose e a possibilidade de desencadear um IAM (LIMA ACS, et al., 2017).

Há na literatura relatos de IAM em mulheres jovens e sem fatores de risco apenas em uso de ACO de baixas dosagens de estrogênio. Nesse contexto, é essencial avaliar os riscos cardiovasculares inerentes daquela mulher e se estes quando combinados ao uso de contraceptivos hormonais combinados podem levar a um evento como IAM, sendo essencial analisar e monitorar cada paciente (ASLAN NA, et al., 2016).

\section{Critérios de elegibilidade da OMS:}

Em 2015, a Organização Mundial de Saúde (OMS) atualizou os Critérios de Elegibilidade para uso de métodos anticoncepcionais. Nessa atualização foram definidas classificações: quando há recursos para julgamento clínico podem ser classificados em quatro categorias: OMS 1 (método deve ser usado); OMS 2 (benefício supera o risco); OMS 3 (risco supera o benefício); e OMS 4 (método não deve ser usado em hipótese alguma). Quando esses recursos clínicos são limitados será classificado em sim (uso o método) e não (não use o método) (OMS, 2015).

Pode-se analisar os critérios para cada morbidade associada ao risco cardiovascular. Em relação às tromboses, os critérios definem: mulheres com história de cirurgia de pequeno porte sem imobilização prolongada como OMS 1; mulheres com história familiar de TVP/TEP ou história de cirurgias de grande porte sem imobilização prolongada como OMS 2; e mulheres com histórico de TVP/TEP ou atual e história de cirurgia de grande porte com imobilização prolongada como OMS 4 (OMS, 2007).

Em relação à hipertensão, mulheres com histórico de hipertensão sem que a PA possa ser avaliada, hipertensão adequadamente controlada ou PA elevada (sistólica 140-159 ou diastólica 90-99) são classificados como categoria OMS 3 e níveis de pressão arterial elevados (sistólica maior ou igual a 160 ou diastólica maior ou igual a 100) são classificados como categoria OMS 4. No caso de mulheres com história de pressão alta durante a gravidez é classificado como OMS 2 (FINOTTI M, 2015).

Nas mulheres diabéticas caso haja história de diabetes gestacional é classificado como OMS 1; diabetes vascular (dependente ou não de insulina) OMS 2; e caso haja danos em órgãos alvo (rins, olhos, nervos), outra doença vascular ou diabetes com mais de 20 anos de duração estão enquadrados nas classificações OMS 3 e 4, dependendo da gravidade do problema. Tanto mulheres com história de IAM ou AVE são classificadas como OMS 4, não podendo fazer o uso de anticoncepcionais orais combinados. Já mulheres com hiperlipidemias conhecidas vão depender do tipo e severidade além da presença de outros fatores de risco cardiovasculares, podendo ser classificadas OMS 2 ou 3 (OMS, 2007).

As mulheres tabagistas o risco será classificado de acordo com a idade: maior ou menor de 35 anos, sendo que nas mais velhas será analisado a quantidade de cigarros: se menos de 15 por dia é classificado como OMS 3 mas caso essa quantidade seja maior ou igual a 15 classificado como OMS 4 (FINNOTI M, 2015).

\section{CONSIDERAÇÕES FINAIS}

O uso do estrogênio exógeno contido nos ACOs, principalmente em altas doses, é um fator preponderante para o desenvolvimento de doenças cardiovasculares. Dessa forma, é necessário um constante acompanhamento da população exposta, ampliando de forma progressiva os conhecimentos acerca dos efeitos adversos e dos riscos potencializados pelo uso desse contraceptivo. Além disso, é fundamental que os profissionais de saúde prescrevam esse método contraceptivo de forma individualizada e segura, visando evitar desfechos desfavoráveis. Ademais, é crucial que ocorra a conscientização acerca da automedicação potencialmente perigosa no uso dos anticoncepcionais hormonais. 


\section{REFERÊNCIAS}

1. ANITA M, SHARON C. Post pregnancy contraception. Best Practice \& Research Clinical Obstetrics \& Gynaecology, 2020; 66: 41-54.

2. ALMEIDA APF, ASSIS MM. Efeitos colaterais e alterações fisiológicas relacionadas ao uso contínuo de anticoncepcionais hormonais orais. Revista Eletrônica Atualiza Saúde, 2017; 5(5): 85-93.

3. ASLAN NA, et al. Low-dose combined oral contraceptives induced acute myocardial infarction. European Journal of Contraception \& Reproductive Health Care, 2016; 21(6): 499-501.

4. BRITTON LE, et al. CE: Na Evidence-Based Update on Contracepcion. American Journal of Nursing, 2020;120(2):2223.

5. CALHOUN AH. Hormonal Contraceptives and Migraine with Aura - Is There Still a Risk? Headache Journal, 2017; 2(57): 184-193.

6. CHARLO PB, et al. Relação entre trombose venosa profunda e seus fatores de risco na população feminina. Glob Acad Nurs, 2020; 1(1): e10.

7. $\mathrm{CHO}$ MK. Use of combined oral contraceptive in perimenopausal women. Chonnam Med Journal, 2018; 54(3): 153158.

8. DRAGOMAN MV, et al. A systematic review and meta-analysis of venous thrombosis risk among users of combined oral contraception. Int J Gynaecol Obstet., 2018 Jun;141(3):287-294.

9. ENEA C, et al. Arterial Stiffness and Hemodynamics in Young women: The effects Of Oral contraceptive Intake and Physical Habits. International Journal of Environmental Research and Public Health, 2021; 18(7): 3392.

10. EVANGELISTA D, et al. Conhecimento e prática anticoncepcional de mulheres portadoras de Diabetes Mellitus. Escola Anna Nery Revista de Enfermagem, 2014; 18(3): 441-447.

11. FERREIRA BBR, DA PAIXÃO JA. A relação entre o uso da pílula anticoncepcional e o desenvolvimento da trombose venosa profunda no Brasil. Revista Artigos.Com, 2021; 29: e7766.

12. FERREIRA LF, et al. O uso da pílula anticoncepcional e as alterações das principais vias metabólicas/ The use of contraceptive pill and alterations of main metabolic pathways. Femina, 2019; 47(7): 426-432.

13. FINOTTI M. Manual de anticoncepção. 1 ed. São Paulo: Federação Brasileira das Associações de Ginecologia e Obstetrícia (FEBRASGO), 2015.

14. GIALERAKI A, et al. Oral Contraceptives and HRT Risk of Thrombosis. Clin Appl Thromb Hemost, 2018; 24(2): 217225.

15. GRAY B, et al. Contraceptive Management for Women Who Are at High Risk of Thrombosis. Clinical Obstetrics and Gynecology, 2018; 61(2): 243-249.

16. GUEDES JVN, et al. Evaluation of lipid profile, high-sensitivity C-reactive protein and D-dimer in users of oral contraceptives of diferents types. Jornal Brasileiro de Patologia e Medicina Laboratorial, 2018; 54(1): 14-20.

17. HUGON-RODIN J, et al. Hormonal tratments and venous thrombosis in women. Ver Med Suisse. $2017 ; 13$ (580): 18211825 .

18. JUREMA K, JUREMA H. Efeitos colaterais a longo prazo associados ao uso de anticoncepcionais hormonais orais. Revista Cereus, 2021; 13(2): 1-12.

19. LEITE C. Saúde cardiovascular das mulheres no século XXI. Brazilian Journal of Development, 2020; 6(11): 9067390687.

20. LIMA ACS, et al. Influência de anticoncepcionais hormonais e ocorrência de acidente vascular cerebral: revisão integrativa. Revista Brasileira de Enfermagem, 2017; 70(3): 675-683.

21. LIU H, et al. Association between duration of oral contraceptive use and risk of hypertension: a meta-analysis. Journal of Clinical Hypertension, 2017; 83(19): 1032-1041.

22. LOPEZ LM, et al. Steroidal contraceptives: effect on carbohydrate metabolism in women without diabetes mellitus (Reviews). Cochrane Data base Systematic Reviews. 2019, 4(5): 5-8.

23. MARJORIBANKS J, et al. Long-term hormone therapy for perimenopausal and postmenopausal women. Cochrane Databases Syst Review, 2017; 1(1).

24. MINISTÉRIO DA SAÚDE. Instituto Sírio-libanês de Ensino e Pesquisa. Protocolos de Atenção Básica: Saúde das Mulheres. $\quad$ Brasil, $2016 . \quad$ Disponível em: https://bvsms.saude.gov.br/bvs/publicacoes/protocolos_atencao_basica_saude_mulheres.pdf. Acessado em: 23 de julho de 2021.

25. MINISTÉRIO DA SAÚDE. Secretaria de Vigilância em Saúde, Departamento de Vigilância de Doenças e Agravos não Transmissíveis e Promoção da Saúde. Saúde Brasil 2018: Uma análise da situação de saúde e das doenças e agravos crônicos: desafios e perspectivas. Brasil, 2018. Disponível em: https://bvsms.saude.gov.br/bvs/publicacoes/saude_brasil_2018_analise_situacao_saude_doencas_agravos_cronico S_desafios_perspectivas.pdf. Acessado em: 09 de junho de 2021.

26. OEDINGEN C, et al. Systematic review and meta-analysis of the association of combined oral contraceptives on the risk of venous thromboembolism: The role of the progestogen type and estrogen dose. Thromb Res., 2018; 165: 6878. 
27. OLIVEIRA RPC, TREVISAN M. O anticoncepcional hormonal via oral e seus efeitos colaterais para as mulheres. Revista Artigos. Com, 2021; 28: e7507.

28. ORGANIZAÇÃO MUNDIAL DA SAÚDE (OMS). Guia de Implantação dos Critérios Médicos de Elegibilidade e das Recomendações da OMS para 0 uso de Contracepção. 2018. Disponível em: http://apps.who.int/iris/bitstream/handle/10665/272758/9789247513573-por.pdf?ua=1. Acessado em: 24 de julho de 2021.

29. ORGANIZAÇÃO MUNDIAL DA SAÚDE (OMS). Medical eligibility criteria for contraceptive use. 2015. Disponível em: http://apps.who.int/iris/bitstream/handle/10665/181468/9789241549158_eng.pdf?sequence=9. Acessado em: 12 de junho de 2021.

30. ORGANIZAÇÃO MUNDIAL DA SAÚDE (OMS). Planejamento familiar: um manual global. 2007. Disponível em: http://apps.who.int/iris/bitstream/handle/10665/44028/9780978856304_por.pdf;jsessionid=11986712F0524CF10F7C CO0FAF41837A?sequence=6. Acessado em: 27 de agosto de 2021 .

31. RAHHAL A, et al. Low dose combined oral contraceptives in duced thrombotic anterior wall myocardial infarction: a case report. BMC Cardiovascular Disorders, 2020; 20(1): 182.

32. RIBEIRO CCM, et al. Effects of different hormonal contraceptives in women's blood pressure values. Revista Brasileira de Enfermagem, 2018; 71(3): 1453-1459.

33. ROACH REJ, et al. Combined oral contraceptives: The risk of my cardial infarction andischemic stroke. Cochrane Datbases of Systematic Reviews, 2015; 3(8): 2-4.

34. RÖMER T. Medical Eligibility for contraceptive in women at increased risk. Dtsch Arztebl, 2019; 116(45): 764-774.

35. SACCO S, et al. Effect of exogenous estrogens and progestogens on the course of migraine during reproductive age: a consensus statement by the European Headache Federation (EHF) and the European Society of Contraception and Reproductive Health (ESCRH). The Journal of Headache na Pain. 2018; 19(1): 76.

36. SITRUCK-WARE R, et al. Contraception technology; past, present and future. Contraception, $2020 ; 87$ (3): $319-330$.

37. SONOU A, et al. Absolute cardiovascular risk of women using hormonal contraception in Porto Novo. Cardiovascular Journal of Africa, 2018; 29(2): 71. 